\title{
Perfil sociodemográfico e sobrecarga de cuidadores informais de pacientes assistidos em ambulatório de terapia ocupacional
}

\author{
Maria Luisa Gazabim Simôes Ballarin, Aline Cristina Benedito, \\ Catherine Amorim Krön, Daniela Christovam
}

Centro de Ciências da Vida, Faculdade de Terapia Ocupacional, Pontifícia Universidade Católica de Campinas - PUC, São Paulo, SP, Brasil.

\begin{abstract}
Resumo: Introdução: Situações que envolvem doenças graves, degenerativas e/ou incapacidades funcionais (motora, sensopercepetiva ou cognitiva) trazem alterações na vida cotidiana, não somente do sujeito adoecido, mas também de todos os envolvidos, incluindo o Cuidador Informal - CI que o acompanha, evidenciando sobrecarga que pode estar diretamente relacionada à tarefa de cuidar. Objetivo: Assim, este estudo teve por objetivo identificar o perfil sociodemográfico e a sobrecarga dos CI que acompanham pacientes assistidos em Ambulatório de Terapia Ocupacional. Método: Trata-se de um estudo exploratório, descritivo, de corte transversal e de natureza quanti-qualitativa. Para seu desenvolvimento foram utilizados dois instrumentos de coleta de dados: a Escala padronizada Zarit Burden Interview - ZBI, que avalia sobrecarga em cuidadores, e um Questionário sociodemográfico que continha perguntas abertas e fechadas, cujas variáveis de gênero, idade, ocupação, estado civil e escolaridade foram analisadas. Resultados: Os resultados obtidos evidenciaram que os cuidadores eram, em sua maioria, mulheres, mães, casadas, católicas, cujas idades variavam de 30 a 50 anos. Além disso, constatou-se que a maioria (46,6\%) apresentou sobrecarga avaliada como moderada, e 43,4\% dos participantes apresentaram sobrecarga classificada como moderada a severa. Conclusão: Por fim, o estudo possibilitou constatar a necessidade e a importância da construção de estratégias mais efetivas que possam ser adotadas no sentido de assistirem integralmente os Cuidadores Informais. Entende-se que os resultados obtidos podem contribuir subsidiando a implantação de ações e programas de treinamento/suporte, melhorando a qualidade da atenção a todos os envolvidos.
\end{abstract}

Palavras-chave: Terapia Ocupacional, Cuidadores, Relações Familiares.

\section{Sociodemographic profile and burden of informal caregivers of patients assisted in occupational therapy outpatient clinic}

\begin{abstract}
Introduction: Situations involving serious, degenerative diseases and/or functional disabilities (motor, sensory-perceptual or cognitive) affect the patient's daily life and all those involved, including the Informal Caregiver - IC that accompanies them, showing a burden that can be directly related to the care task. Objective: This study aimed to identify the sociodemographic profile and burden of ICs accompanying patients in the Occupational Therapy Outpatient Clinic. Method: This is an exploratory, descriptive, cross-sectional of quantitative and qualitative nature. We used two data collection instruments, the standardized scale, Zarit Burden Interview - ZBI assessing the burden of caregivers and a sociodemographic questionnaire that contained open and closed questions, which variables: gender, age, occupation, marital status, among others, were analyzed. Results: The results showed that caregivers were mostly women, mothers, married, catholic, between 30 to 50 years. Furthermore, it was found that most (46.6\%) rated as moderate burden presented, followed by $43.4 \%$ rated as moderate to severe burden. Conclusion: Finally, the study allowed to verify the necessity and the importance of building more effective strategies that can be adopted to fully attend the Informal Caregivers. These results may contribute subsidizing the implementation of actions and training programs/support improving the quality of care of everyone involved.
\end{abstract}

Keywords: Occupational Therapy, Caregivers, Family Relations.

Autor para correspondência: Maria Luisa Gazabim Simões Ballarin, Centro de Ciências da Vida, Faculdade de Terapia Ocupacional, Pontifícia Universidade Católica de Campinas, Av. John Boyd Dunlop, s/n, Jardim Ipaussurama, CEP 13060-904, Campinas, SP, Brasil, e-mail: mlballarin@puc-campinas.edu.br

Recebido em Dez. 17, 2014; $1^{\text {a }}$ Revisão em Maio 7, 2015; $2^{\text {a }}$ Revisão em Jul. 29, 2015; $3^{\text {a }}$ Revisão em Ago. 18, 2015 ; $4^{\text {a }}$ Revisão em Out. 3 , 2015; Aceito em Out. 24, 2015. 


\section{Introdução}

O termo Cuidador Informal - CI designa comumente um membro da família ou não, que assumiu a tarefa de cuidar, exercendo todos os cuidados necessários a uma pessoa com alguma patologia ou deficiência, incluindo cuidados higiênicos, alimentícios, de locomoção, financeiros, medicamentosos, entre outros, sem remuneraçáo e sem possuir formação profissional específica e/ou preparo técnico para desempenhar as atividades de caráter assistencial, caracterizando-se como figura principal no acompanhamento do paciente que, no caso, apresenta incapacidades temporárias ou definitivas.

$\mathrm{Na}$ atualidade, os cuidadores informais vêm sendo considerados recursos a serviço da pessoa adoecida, mas não necessariamente foco de atenção da equipe de saúde. Nesse sentido, faz-se necessário enfatizar a importância de seu papel, considerando-o também como uma pessoa que pode estar necessitando de auxílio e apoio para o desempenho de sua funçáo de cuidar e, portanto, sujeito merecedor de atenção e cuidado dos profissionais de saúde (ARAÚJO et al., 2009).

Floriani e Schramm (2006) e Beck e Lopes (2007) apontam para o fato de que o CI deve receber suporte não apenas para aprender a cuidar do paciente, mas, sobretudo, para enfrentar, compreender e compartilhar a situação de doença e/ou deficiência, para que possa lidar mais adequadamente com seus próprios problemas, conflitos, medo e aumento das responsabilidades, principalmente, por se observar que CI de doentes crônicos apresentam prejuízos alarmantes.

Os estudos sobre o perfil sociodemográfico de cuidadores informais evidenciam tratar-se, na maioria das vezes, de mulheres, casadas, que convivem com o paciente no próprio domicílio. Além disso, não exercem atividades remuneradas e possuem grau de parentesco com o paciente que assistem. Desempenham a função de cuidar de pessoas que apresentam diferentes problemas de saúde, tais como: doença pulmonar crônica obstrutiva, câncer em estado terminal, Alzheimer, paralisia cerebral, entre outras. Na maioria das vezes, esses problemas de saúde acarretam incapacidades, tornando os pacientes parcial ou totalmente dependentes (ARAUJO et al., 2009; BECK; LOPES, 2007; DOMINGUES; SANTOS; QUINTANS, 2009; YAMASHITA et al., 2010).

Os cuidados cotidianos dispensados pelos cuidadores informais ao paciente envolvem o engajamento em inúmeras e extensas atividades, por isso mesmo, Floriani (2004) descreve que os cuidadores vêm manifestando sintomas como: estresse, depressão, distúrbios do sono, entre outros, evidenciando sobrecarga.

Fatores como a gravidade da doença, o nível de incapacidade funcional, o comprometimento na habilidade de comunicar-se, o número de medicamentos em uso, a presença de distúrbios psiquiátricos, visuais, auditivos e/ou cognitivos apresentados pelos pacientes estáo associados ao nível de sobrecarga e à qualidade de vida do CI. Assim, segundo alguns autores, quanto mais dependente $\mathrm{e}$ desfavorável forem as condiçóes dos pacientes, maior será o impacto e as atribuiçóes relativas ao ato de cuidar, desempenhadas pelo CI (WASSERMAN, 1992; COMARU; MONTEIRO, 2008).

A sobrecarga do CI pode acarretar problemas físicos, psicológicos, emocionais, sociais e financeiros, que acabam afetando tanto o bem-estar do cuidador como do paciente. Assim, é evidente que as atribuiçóes do CI, pertinentes ao ato de cuidar de seu familiar adoecido e/ou dependente, interferem nos aspectos de sua própria vida pessoal, familiar, laboral e social, predispondo-os a conflitos (ARAUJO; PAUL; MARTINS, 2009; ARAUJO et al., 2013).

Embora o conceito de sobrecarga seja abrangente e complexo, frequentemente está associado ao sentido negativo do termo, pois as açóes de cuidado e seus impactos na vida dos cuidadores são descritos como uma "carga", termo que etimologicamente associa-se à ideia de "peso", ou "o que pesa sobre uma pessoa", "incumbência” (RICARTE, 2009). A sobrecarga refere-se ao desgaste do CI por cansaço, traduzindo-se em função de fatores de estresse significativos e um espaço de tempo curto ou a uma exposiçáo prolongada a fatores de estresse pouco significativos, mas que ao longo do tempo originam consequências (SEQUEIRA, 2010).

De modo geral, pode-se dizer que a sobrecarga engloba a esfera biopsicossocial e resulta da busca de um equilíbrio entre diferentes variáveis, tais como: disponibilidade de tempo para realização do cuidado, recursos financeiros, condiçóes psicológicas, físicas e sociais, atribuiçôes e distribuição de papéis (OLIVEIRA et al., 2012).

Com base nos aspectos descritos, o presente estudo buscou identificar o perfil sociodemográfico e a sobrecarga de cuidadores informais que acompanhavam pacientes em tratamento no Ambulatório de Terapia Ocupacional. Entende-se que os resultados obtidos podem subsidiar o planejamento de açóes que atendam às necessidades de saúde dos cuidadores informais e minimizem o impacto que a função de cuidar pode acarretar em seus cotidianos, melhorando a qualidade da atenção de todos os envolvidos. 


\section{Método}

Trata-se de um estudo descritivo, exploratório, de corte transversal e de natureza quanti-qualitativa. Participaram do estudo 30 cuidadores informais que acompanhavam pacientes (crianças e adultos) atendidos em Ambulatório de Terapia Ocupacional. Os critérios de inclusão estabelecidos para seleção dos sujeitos de investigaçáo contemplaram o fato de serem cuidadores informais, com idade igual ou superior a 18 anos, de ambos os sexos, que estavam realizando a tarefa de cuidar por um período mínimo de seis meses, que não recebiam remuneração para o desempenho da tarefa de cuidar e que aceitassem participar do estudo, assinando o Termo de Consentimento Livre e Esclarecido - TCLE. Foram excluídos do estudo 06 cuidadores, dos quais 04 não concordaram em participar da pesquisa e 02 cuja função de CI não foi possível identificar, pois embora se assumissem como cuidadores informais, acompanhavam esporadicamente seus familiares no serviço e, quando inquirido sobre as atividades de cuidado desenvolvidas para e com o seu familiar, não as caracterizavam como atividades rotineiras, ou seja, realizadas com frequência.

Para a realizaçáo da coleta de dados no campo foram utilizados dois instrumentos de investigação, a Escala padronizada Zarit Burden Interview -ZBI, que avalia sobrecarga em cuidadores, e um Questionário sociodemográfico que continha perguntas abertas e fechadas, cujo foco dirigiu-se a variáveis como sexo, idade, estado civil, escolaridade, crença religiosa, grau de parentesco com o usuário do serviço, tempo de desempenho em relaçáo à função de cuidador, quais eram as atividades e tarefas cotidianas que realizavam para si e para os cuidados necessários ao familiar adoecido.

A escala ZBI é composta de 22 itens que avaliam a relação cuidador-paciente, a condição de saúde, o bem-estar psicológico, finanças e vida social, englobando aspectos objetivos e subjetivos. As informações incluem quatro fatores: o impacto da prestação do cuidado, a relação interpessoal, expectativa frente ao cuidado e percepção de autoeficácia. A escala de respostas contempla uma pontuação que varia de 0 a 4 , de acordo com presença ou intensidade de uma resposta afirmativa $(0=$ nunca; $1=$ raramente; $2=$ =lgumas vezes; 3 =frequentemente; $4=$ sempre), exceto no último item da Escala (no qual o entrevistado é questionado se está se sentindo sobrecarregado no papel de cuidador). Para este último item da Escala, as respostas são pontuadas considerando-se $(0=$ nem um pouco $; 1=$ um pouco; $2=$ moderadamente; $3=$ muito; $4=$ extremamente). $\mathrm{O}$ escore total da escala varia de 0 a 88; quanto maior o escore, maior a sobrecarga (FERREIRA et al., 2010; SCAVUFCA, 2002). Assim, a sobrecarga dos cuidadores pode ser classificada de acordo com a Tabela 1 .

Após a aprovação do estudo pelo Comitê de Ética em Pesquisa (protocolo no 0489/11), procedeu-se à coleta de dados no campo ocorrida entre os meses de agosto de 2013 e março de 2014. Inicialmente, os cuidadores informais foram contatados no Ambulatório de Terapia Ocupacional, serviço vinculado à Faculdade de Terapia Ocupacional de uma Universidade do interior do Estado de Sáo Paulo que presta atendimentos aos pacientes conveniados do Sistema Único de Saúde - SUS, sendo seu objetivo recuperar, habilitar e restaurar indivíduos que apresentam diferentes problemáticas que acabam comprometendo, em algum nível, a funcionalidade e/ou a realização das atividades cotidianas. Neste contato inicial, o possível sujeito de investigação (cuidador informal) era informado sobre os objetivos da pesquisa e convidado a participar do estudo. Com a concordância voluntária do CI, dois encontros subsequentes eram agendados para efetivar a coleta de dados.

Enfatiza-se que os dados coletados subsidiaram a elaboração de um banco de dados construído a partir de uma planilha no Excel e, posteriormente, receberam tratamento estatístico descritivo simples. Quanto aos dados qualitativos, resultantes das questôes abertas contidas no Questionário, foram analisados com base nos pressupostos teóricos relativos à Análise do Discurso - AD.

A análise propriamente é realizada pela leitura exaustiva do material, para explorá-lo e construir o tratamento e a interpretação dos dados. Neste momento, o pesquisador identifica o contexto da produçáo do discurso para codificá-lo, identificar suas unidades de registros, bem como as categorias que dele emergem (MACEDO et al., 2008, p. 652).

O tratamento dos dados exigiu etapa de pré-análise do material obtido, seguida da seleção dos dados, considerando a articulação destes com os objetivos estabelecidos e com os referenciais teóricos que fundamentam este estudo.

Tabela 1. Classificação do nível de sobrecarga Escores da Escala ZBI.

\begin{tabular}{cl}
\hline Escore & \multicolumn{1}{c}{ Nível de Sobrecarga } \\
\hline$<\mathbf{2 1}$ & Ausência ou pouca sobrecarga \\
$\mathbf{2 1}$ e 40 & Sobrecarga moderada \\
41 a 60 & Sobrecarga moderada a severa \\
61 a 88 & Sobrecarga severa \\
\hline
\end{tabular}




\section{Resultados e Discussão}

A análise dos resultados obtidos evidenciou diversidade tanto em relação ao perfil sociodemográfico como em relação às atividades desempenhadas e o nível de sobrecarga dos cuidadores informais investigados.

\subsection{Perfil sociodemográfico e a rotina de atividades dos cuidadores informais}

Da amostra estudada, observou-se que os cuidadores eram predominantemente mulheres $(77 \%)$, com idades que variaram de 30 a 50 anos (média de idade igual a 44,5 anos), casadas (63\%) e mães de crianças assistidas no referido serviço (67\%), dados que corroboram os estudos de Silva et al. (2006) e Araújo, Paul e Martins (2009). Além disso, a maioria dos cuidadores residia na mesma moradia que o familiar (89\%) e não dividia a tarefa do cuidado com outra pessoa $(72,5 \%)$.

Em relação à escolaridade dos cuidadores, foi possível observar que $26 \%$ não havia completado o $1^{\circ}$ grau, $33 \%$ havia concluído o $2^{\circ}$ grau e apenas $3,3 \%$ possuía nível superior, dados que confirmam os descritos por Nakatani et al. (2003). Embora neste estudo a correlaçáo existente entre o grau escolaridade e o nível de sobrecarga dos cuidadores não tenha sido objeto de análise, enfatiza-se que esta correlaçáo tem sido descrita na literatura de modo divergente. Araujo et al. (2013) referem que cuidadores com escolaridade elevada são afetados com os mesmos problemas que os cuidadores com nível de escolaridade mais baixa; já para Tabeleão, Tomasi e Quevedo (2014), a baixa escolaridade do CI pode implicar dificuldade do cuidador em assimilar informaçóes e conhecimento sobre a doença, fator que afeta diretamente sua sobrecarga.

Quanto à rotina de atividades e tarefas que desenvolviam, constatou-se que $70 \%$ se dedicava às atividades do lar, como limpar a casa, fazer almoço, lavar roupa, ir ao supermercado, além das atividades de cuidado, como dar medicação, acompanhar o familiar aos serviços de saúde, cuidar da alimentação e higiene, transferência para o vaso sanitário e cama. Constatou-se ainda que a maioria dos cuidadores (66\%) havia se desligado do emprego para se dedicar integralmente aos cuidados de seus familiares, pois suas rotinas estavam envoltas em tarefas que eram realizadas em circunstâncias diferentes das usuais. As tarefas exigiam mais tempo para serem realizadas do que o normal e requeriam manejos diferenciados, como por exemplo: alimentação por meio de sonda, locomoção assistida-empurrar cadeira de roda, cuidados quanto à mudança de decúbito, manutenção de dispositivos e órteses, realização de banho em leito, etc. Ressalta-se que os familiares acompanhados pelos cuidadores investigados apresentavam prioritariamente quadros de Paralisia Cerebral - PC (33,4\%), Lesão Medular - TRM (16,6\%) e Trauma Cranioencefálico - TCE (13,4\%), sendo a maioria dependente ou parcialmente dependente na realização das Atividades de Vida Diária - AVD.

Os resultados descritos evidenciam que, de fato, historicamente as mulheres vêm assumindo o papel de provedora de cuidados gerais da família, enquanto ao homem cabe a função do sustento material. Esses dados reafirmam aspectos relacionados ao gênero e grau de parentesco, ou seja, por serem a maioria mulheres e mães, elas não conseguem ter outras funçôes extradomiciliares e acabam saindo do emprego. Assim, exercem em tempo integral o cuidado, fazendo com que apenas o pai ou companheiro seja o provedor principal do sustento (OLIVEIRA; PEREIRA; FREITAS, 2010).

Gonçalves et al. (2006) e Milbrath et al. (2008) afirmam que o cotidiano e a autoestima dessas mães são os aspectos mais afetados decorrentes dessa dependência financeira, pois culturalmente as mães se veem diante de atribuiçôes sobre as quais recai a responsabilidade do ato de cuidar, a crença de que náo existe outra pessoa capaz de suprir as necessidades do filho (superproteção) e o vínculo sanguíneo e afetivo (BECK; LOPES, 2007; CAMARGOS et al., 2009; MARTINS; SOLER, 2008; OLIVEIRA; PEREIRA; FREITAS, 2010). Com isso, o suporte emocional, afetivo e de cuidado fica a cargo da mulher. Entretanto, é importante salientar que, atualmente, esses papéis vêm sofrendo alteraçôes (CAMARANO; PASINATO; LEMOS, 2007; PIMENTA et al., 2009).

Constatou-se ainda que $77 \%$ dos cuidadores referiram ter deixado em segundo plano suas próprias vidas, negligenciando muitas vezes atividades de autocuidado, lazer e/ou sociais, resultado que coincide com os obtidos por Fonseca, Penna e Soares (2008). Evidencia-se, desta forma, que o aumento das responsabilidades assumidas pelos cuidadores informais interfere em suas vidas, pois suas açóes diárias passam a se voltar para o familiar a ser cuidado.

Quanto à religião, constatou-se que 57\% eram católicas e 40\% evangélicas, dados que corroboram com os de outros estudos. Segundo Santos e Cunha 
(2013) e Simonetti e Ferreira (2008), as crenças religiosas servem como facilitadores na aceitação, resignação e conformismo do quadro patológico do familiar, além de intermediarem o enfrentamento do cuidado diário e integral. De Nardi et al. (2011) e Vilela et al. (2006) referem que cuidadores que náo possuem apoio religioso apresentam maior índice de adoecimento, já que vivenciam rotinas excessivamente sobrecarregadas.

Com relação ao tempo em que vinham desempenhando a função de cuidar, constatou-se que $53 \%$ exerciam esta função há três ou quatro anos. A correlação existente entre o tempo de desempenho da funçáo de cuidar e a sobrecarga vem sendo abordada em alguns estudos (SANTOS; CUNHA, 2013; VALENTE et al., 2013; ZAMBRANODOMINGUEZ; GUERRA-MARTIN, 2012), embora, neste estudo, esta correlação não tenha sido objeto de análise.

\subsection{Sobrecarga dos cuidadores informais}

Constatou-se que os cuidadores deste estudo apresentaram níveis de sobrecarga variados, conforme ilustra a Figura 1.

Os resultados obtidos demonstraram que a maioria $(46,6 \%)$ dos cuidadores investigados apresentou sobrecarga avaliada como moderada, seguido de $43,4 \%$ de sobrecarga classificada como moderada a severa. $\mathrm{O}$ total da média dos escores finais da ZBI neste grupo de cuidadores foi de 41,2 (0-88), indicando sobrecarga moderada a severa. Tais resultados coincidiram com os descritos em outros estudos que também objetivaram avaliar a sobrecarga de cuidadores de crianças e adultos a partir da Escala ZBI (AMENDOLA, 2007; AMENDOLA; OLIVEIRA; ALVARENGA, 2008; CAMARGOS et al., 2009; MASLACH;

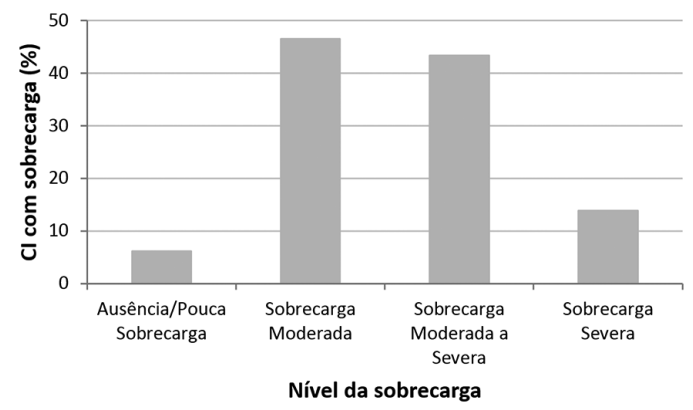

Figura 1. Distribuição percentual do nível de sobrecarga dos cuidadores investigados.
SCHAUFELI; LEITER, 2001; WACHHOLZ; SANTOS; WOLF, 2013).

Constatou-se que $73 \%$ dos cuidadores tinham a sensação de que eram as únicas pessoas de quem o familiar podia depender e $66 \%$ disseram sentir-se estressados por cuidarem de seu familiar e, ao mesmo tempo, desempenharem as outras responsabilidades com a família. Além disso, $63 \%$ sentia que sua vida social havia sido afetada em função do envolvimento com o familiar dependente de cuidado e $59 \%$ referiram ter a sensação de não ter dinheiro suficiente para cuidar do familiar e das despesas cotidianas, dados que confirmam os descritos por Amendola (2007).

Verificou-se também que, quando os cuidadores foram inquiridos sobre a própria percepção acerca do nível de sobrecarga, $53 \%$ disseram não se sentirem sobrecarregados, $23 \%$ só um pouco, $12 \%$ moderadamente, $6 \%$ muito e $6 \%$ extremamente. Embora a maioria dos cuidadores (53\%) refira não se sentir sobrecarregado, ao se observar sobrecarga identificada por meio do ZBI, constata-se que $90 \%$ apresentam níveis classificados entre moderado e moderado a severo, evidenciando distanciamento entre a sobrecarga percebida e a aferida. A dificuldade do cuidador em perceber e assumir diretamente a sobrecarga vivenciada foi apontada nos estudos de Santos et al. (2010) e Amaral et al. (2011), que atribuíram este distanciamento à interferência de aspectos como medo, receio e sentimentos de vergonha do $\mathrm{CI}$ ao responder sobre sua relação com a tarefa de cuidar.

Como se pode constatar, a análise dos resultados obtidos neste estudo, pertinentes à aferição de sobrecarga, é indicativa tanto de sobrecarga objetiva como subjetiva. A sobrecarga relacionada ao cuidado é uma condição de risco à qual estão expostos cotidianamente os familiares que desempenham o papel de cuidador, sendo de fundamental importância o planejamento e a implementação de açóes de saúde no serviço em que estes acompanham seus respectivos familiares/pacientes, para que, assim, possam ser orientados e recebam o suporte necessário pertinente para a realização das atividades cotidianas inerentes ao cuidado, minimizando a sobrecarga por eles vivenciada (GREENWOOD et al., 2008).

\section{Conclusão}

Depreende-se do estudo desenvolvido que a maioria dos cuidadores informais investigados apresentou sobrecarga avaliada como moderada e moderada a severa, evidenciando que estáo expostos a fatores de risco e de adoecimento. Tais resultados devem ser analisados com prudência, pois as limitaçóes 
do estudo devem ser consideradas, uma vez que contemplou amostra reduzida de cuidadores de um único serviço de saúde. Apesar disso, o estudo evidencia alguns aspectos que permeiam a rotina e as atividades desempenhadas pelos cuidadores e que podem estar relacionadas ao aumento da sobrecarga destes.

Assim, torna-se relevante o desenvolvimento de estudos mais detalhados sobre o tema, bem como a ampliação das açôes de saúde que tomem o cuidador como sujeito-alvo de intervenção. Neste sentido, o estudo aponta para a necessidade de a equipe de profissionais do serviço implementar e oferecer açóes de apoio, treinamento e orientação aos cuidadores, de modo a auxiliá-los na realização das atividades de cuidado, minimizando assim a sobrecarga vivenciada por eles e contribuindo para uma relaçáo de cuidado, caracterizada pela preocupação com a integralidade e a singularidade do ser humano e pela valorização da relação e do respeito ao outro.

\section{Referências}

AMARAL, E. G. et al. Qualidade de vida e sobrecarga em cuidadores de crianças com Síndrome de Down. Revista Movimenta, Goiânia, v. 4, n. 2, p. 99-108, 2011.

AMEDOLA, F.; OLIVEIRA, M. A. C.; ALVARENGA, M. R. M. Qualidade de vida dos cuidadores de pacientes dependentes no programa Saúde da Família. Texto e contexto enfermagem, Florianópolis, v. 7, n. 2, p. 266-272, 2008.

AMENDOLA, F. Qualidade de vida de cuidadores familiares de pacientes com perdas funcionais atendidos pelo Programa saúde da família, do município de São Paulo. 2007. 166 f. Dissertação (Mestrado em Enfermagem) Universidade de São Paulo, São Paulo, 2007.

ARAÚJO, I. M.; PAUL, C.; MARTINS, M. M. Cuidar de idosos dependentes no domicílio: desabafos de quem cuida. Ciência, Cuidado e Saúde, Maringá, v. 8, n. 2, p. 191-197, 2009.

ARAUJO, J. S. et al. Perfil dos cuidadores e as dificuldades enfrentadas no cuidado ao idoso, em Ananindeua, PA. Revista brasileira geriatria gerontologia, Rio de Janeiro, v. 16, n. 1, p. 149-158, 2013.

ARAÚJO, L. Z. S. et al. Cuidador principal de paciente oncológico fora da possibilidade de cura, repercussóes deste encargo. Revista Brasileira de Enfermagem, Brasília, v. 62, n. 1, p. 32-37, 2009.

BECK, A. R. M.; LOPES, M. H. B. M. Cuidadores de crianças com câncer: aspectos da vida afetados pela atividade de cuidador. Revista Brasileira de Enfermagem, Brasília, v. 60, n. 6, p. 670-675, 2007.

CAMARANO, A. A.; PASINATO, M. T.; LEMOS, V. R. Cuidados de longa duração para a população idosa: uma questão de gênero? In: NERI, A. L. (Org.). Quali- dade de vida na velhice: enfoque multidisciplinar. Campinas: Alínea, 2007. p. 127-150.

CAMARGOS, A. C. R. et al. Avaliação da sobrecarga do cuidador de crianças com paralisia cerebral através da escala Burden Interview. Revista Brasileira de Saúde Materno Infantil, Recife, v. 9, n. 1, p. 31-37, 2009.

COMARU, N. R. C.; MONTEIRO, A. R. M. O cuidado domiciliar à criança em quimioterapia na perspectiva do cuidador familiar. Revista Gaúcha de Enfermagem, Porto Alegre, v. 29, n. 3, p. 423-30, 2008.

DE NARDI, T. et al. Sobrecarga e percepçáo de qualidade de vida em cuidadores de idosos do Núcleo de Atendimento à Terceira Idade do Exército (Natiex). Revista Brasileira de Geriatria e Gerontologia, Rio de Janeiro, v. 14, n. 3, p. 511-519, 2011.

DOMINGUES, M. A. R. C.; SANTOS, C. F.; QUINTANS, J. R. Doença de Alzheimer: o perfil dos cuidadores que utilizam o serviço de apoio telefônico da ABRAz - Associaçáo Brasileira de Alzheimer. O Mundo da Saúde, São Paulo, v. 33, n. 1, p. 161-169, 2009.

FERREIRA, F. et al. Validação da escala de Zarit: sobrecarga do cuidador em cuidados paliativos domiciliários, para população portuguesa. Cadernos de Saúde, Portugal, v. 3, n. 2, p. 13-19, 2010.

FLORIANI, C. A. Cuidador familiar: sobrecarga e proteção. Revista Brasileira de Cancerologia, Rio de Janeiro, v. 50, n. 4, p. 341-345, 2004.

FLORIANI, C. A.; SCHRAMM, F. R. Cuidador do idoso com câncer avançado: um ator vulnerado. Cadernos de Saúde Pública, Rio de Janeiro, v. 22, n. 3, p. 527534, 2006.

FONSECA, N. R.; PENNA, A. F. G.; SOARES, M. P. G. Ser cuidador familiar: um estudo de caso sobre as consequências de assumir este papel. Physis, Rio de Janeiro, v. 18, n. 4, p. 727-743, 2008.

GONÇALVES, L. H. T. et al. Perfil da família cuidadora de idoso doente/fragilizado do contexto sociocultural de Florianópolis, SC. Texto \& Contexto Enfermagem, Florianópolis, v. 15, n. 4, p. 570-577, 2006.

GREENWOOD, N. et al. Informal carers of stroke survivors: factors influencing carers: a systematic review of quantitative studies. Desability and Rehabilitation, London, v. 30, n. 18, p. 1329-1349, 2008.

MACEDO, L. C. et al. Análise do discurso: uma reflexão para pesquisar em saúde. Interface - Comunicação, Saúde, Educação, Botucatu, v. 12, n. 26, p. 649-657, 2008.

MARTINS, G.; SOLER, Z. A. S. G. Perfil dos cuidadores de crianças com bexiga neurogênica. Arquivos de ciências da saúde, São José do Rio Preto, v. 15, n. 1, p. 13-16, 2008.

MASLACH, C.; SCHAUFELI, W. B.; LEITER, M. P. Job burnout. Annual Review of Psychology, California, v. 52, p. 397-422, 2001. 
MILBRATH, V. M. et al. Ser mulher e mãe de uma criança portadora de paralisia cerebral. Acta Paulista de Enfermagem, São Paulo, v. 21, n. 3, p. 427-431, 2008.

NAKATANI, A. Y. K. et al. Perfil dos cuidadores informais de idosos com déficit de autocuidado atendidos pelo Programa de Saúde da Família. Revista Eletrônica de Enfermagem, Goiás, v. 5, n. 1, p. 15-20, 2003.

OLIVEIRA, A. R. S. et al. Escalas para avaliação de sobrecarga de cuidadores de pacientes com acidente vascular encefálico. Revista Brasileira de Enfermagem, Brasília, v. 65 , n. 5 , p. $839-843,2012$.

OLIVEIRA, D. M. P.; PEREIRA, C. U.; FREITAS, Z. M. P. Conhecimento do cuidador de crianças com hidrocefalia. Revista Brasileira de Enfermagem, Brasília, v. 63, n. 5, p. 782-785, 2010.

PIMENTA, G. M. F. et al. Perfil do familiar cuidador de idoso fragilizado em convívio doméstico da grande Região do Porto, Portugal. Revista da Escola de Enfermagem da USP, São Paulo, v. 43, n. 3, p. 609-614, 2009.

RICARTE, L. F. C. S. Sobrecarga do cuidador informal de idosos dependentes no Concelho da Ribeira Grande. 2009. 134 f. Dissertação (Mestrado em Ciências de Enfermagem) - Universidade do Porto, Portugal, 2009.

SANTOS, A. A. S. et al. Avaliação da sobrecarga dos cuidadores de crianças com paralisia cerebral. Ciência, Cuidado e Saúde, Maringá, v. 9, n. 3, p. 503-509, 2010.

SANTOS, G. S.; CUNHA, I. C. K. O. Perfil sociodemográfico de cuidadores familiares de idosos residentes em uma área de abrangência da Estratégia Saúde da Família no município de São Paulo. Saúde Coletiva, São Paulo, v. 10, n. 60, p. 47-52, 2013.

SCAVUFCA, M. Versão brasileira da escala Burden Interview para avaliação de sobrecarga em cuidadores de indivíduos com doenças mentais. Revista Brasileira de Psiquiatria, São Paulo, v. 24, n. 1, p. 12-17, 2002.

SEQUEIRA, C. A. C. Adaptação e validação da Escala de Sobrecarga do Cuidador de Zarit. Revista de Enfermagem Referência, Coimbra, v. 2, n. 12, p. 9-16, 2010.
SILVA, L. W. S. et al. Perfil da família cuidadora de idoso doente/fragilizado do contexto sociocultural de Florianópolis, SC. Texto \& Contexto Enfermagem, Florianópolis, v. 15, n. 4, p. 570-577, 2006.

SIMONETTI, J. P.; FERREIRA, J. C. Estratégias de coping desenvolvidas por cuidadores de idosos portadores de doença crônica. Revista da Escola de Enfermagem da USP, São Paulo, v. 42, n. 1, p. 19-25, 2008.

TABELEÃO, V. P.; TOMAS, E.; QUEVEDO, L. A. Sobrecarga de familiares de pessoas com transtorno psíquico: níveis e fatores associados. Archives of Clinical Psychiatry, São Paulo, v. 41, n. 3, p. 63-66, 2014.

VALENTE, L. et al. Auto percepção de saúde em cuidadores familiares e o tipo de demência: resultados preliminares de uma amostra ambulatorial. Revista Brasileira de Neurologia, Rio de Janeiro, v. 49, n. 1, p. 13-19, 2013.

VILELA, A. B. A. et al. Perfil do familiar cuidador de idoso doente e/ou fragilizado do contexto sociocultural de Jequié-BA. Revista Brasileira de Geriatria e Gerontologia, Rio de Janeiro, v. 9, n. 1, p. 55-69, 2006.

WACHHOLZ, P. A.; SANTOS, R. C. C.; WOLF, L. S. P. Reconhecendo a sobrecarga e a qualidade de vida de cuidadores familiares de idosos frágeis. Revista Brasileira de Geriatria e Gerontologia, Rio de Janeiro, v. 16, n. 3, p. 513-526, 2013

WASSERMAN, M. D. A. Princípios de tratamento psiquiátrico de crianças e adolescentes com doenças físicas. In: GARFINKEL, B. D.; CARLSON, G. A.; WELLER, E. B. (Org.). Transtornos psiquiátricos na infância e adolescência. Porto Alegre: Artes Médicas, 1992. p. 408-416.

YAMASHITA, C. H. et al. Perfil sociodemográfico de cuidadores familiares de pacientes dependentes atendidos por uma unidade de saúde da família no município de São Paulo. O Mundo da saúde, São Paulo, v. 34, n. 1, p. 20-24, 2010.

ZAMBRANO-DOMINGUEZ, E. M.; GUERRA-MARTIN, M. D. Formação do cuidador informal: relaçáo com o tempo de cuidado de pessoas dependentes maiores de 65 anos. Aquichán, Colombia, v. 12, n. 3, p. 241-251, 2012.

\section{Contribuição dos Autores}

Maria Luisa Gazabim Simões Ballarin: elaboração e orientação do projeto, análise dos dados e redação do artigo. Aline Cristina Benedito, Catherine Amorim Kröne Daniela Christovam, coleta e análise dos dados e redação preliminar do artigo. Todas as autoras aprovaram a versão final do texto. 